\title{
A computerised diagnostic index for medical and neuro-ophthalmology
}

\author{
W H Woon, D J Spalton
}

\begin{abstract}
We have developed a classification that meets the requirements of the Medical Eye Unit and computerised it. The classification uses three categories: anatomical site of lesion, pathological process, and clinical diagnosis. Each patient is identified by standard data and up to three entries in each of anatomical localisation, pathological diagnosis, and clinical diagnosis. This system allows the formation of groups of patients which can be either very specific or very broad by appropriate combination of selection criteria in the different categories. No codes are used but computer validation ensures consistent nomenclature. Computerisation allows rapid searching of records even with multiple selection criteria. The program is simple to use and validates data entries. An additional benefit is that it facilitates medical audit of the department's work.
\end{abstract}

Computerised data bases greatly facilitate the retrieval and correlation of information. ${ }^{\prime}$ However, their usage and practicability very much depend on having a satisfactory classification system of data entry as well as a computer system that is user friendly.

Problems of classification are a particular problem in medical and neuro-ophthalmology, where patients may have rare and esoteric diagnoses or several diseases. A simple listing of clinical diagnoses is inadequate, as patients may have multiple diagnoses - for example, a patient with cryptococcal meningitis, raised intracranial pressure, and occipital cortical infarct - or a diagnosis may be described in several ways - for example, diabetic third nerve palsy, microvascular third nerve palsy, pupil sparing third nerve palsy - or a firm diagnosis may be difficult to establish with certainty. An additional problem with a simple listing of clinical diagnoses is that it is difficult to compile retrospective series of diagnostic, anatomical, or pathological entities such as all third nerve palsies, a review of all orbital disease, or all cases of genetically associated optic atrophy.

There have been modifications of the International Classification of Disease Code (ICD) ${ }^{2}$ for ophthalmic use, and these have been computerised. An example of such a code is the Wilmer Information System (WIS). ${ }^{34}$ The WIS code is designed to identify patients by diagnosis or procedure, and to allow extension for further subclassification. However, the structure of the ICD does not meet our requirements. The hierarchial structure of the ICD and its classification mainly along anatomical lines allows easy formation of broader anatomical groups. The code does not allow easy arrangement of patients into different groups for example, groups classified according to pathological process rather than anatomical site of lesion. We have decided to classify patients by three categories: anatomical site of lesion, pathological process, and clinical diagnosis. We have also decided to avoid a code system altogether, as relational databases allow checking of the diagnoses entered to make sure that they agree with a standard nomenclature. In essence the standard nomenclature has become the code. This system means more typing for the user but saves the user looking up a code, and it is probably less intimidating to the infrequent user.

A classification system using more than one means of classification needs to be computerised, as selecting outpatients on a combination of selection criteria would be very arduous by hand. Problems of computerisation are that the system must be accessible to people who may be with the department for only a short period and who may have no prior knowledge of computers or the classification system used. The system must ensure that data are entered in a consistent manner, otherwise data retrieval will be incomplete. This can be achieved by having files of appropriate entries against which the computer can check data input.

\section{Classification}

Standard data on all patients are entered for personal identification. Patients are then classified by anatomical localisation, pathological diagnosis, and clinical diagnosis. In each of these three classes three listings are possible to allow for multiple diagnoses. Table I shows the data that may be entered for each patient. For the use of the medical eye department the alternatives for anatomical localisation and pathological
Medical Eye Unit, St Thomas's Hospital, London SE1 7EH W H Woon D J Spalton Correspondence to: Mr D J Spalton, Medical Eye Unit, St Thomas's Hospital, London SEl 7EH

Accepted for publication 26 October 1989
TABLE I: Data which may be entered for each patient

\begin{tabular}{|c|c|c|c|c|c|c|}
\hline Name & Hosp No & Age & Sex & Consultant & Date of Admission & District \\
\hline Anat $\mathrm{Lo}$ & \multicolumn{4}{|c|}{ Path Diagnosis } & \multicolumn{2}{|c|}{ Clinical Diagnosis } \\
\hline
\end{tabular}


TABLE II The allowed entries for anatomical localisation and pathological diagnosis

\begin{tabular}{ll}
\hline Anatomical localisation & Pathological diagnoses \\
\hline Cutaneous (eyelids, etc.) & Degenerative \\
Ocular & Demyelination \\
Optic disc & Dystrophy \\
Optic nerve & Genetic \\
Chiasm & Infection \\
Optic tract & Inflammatory \\
Cortical & Raised intracranial pressure \\
Orbit & Trauma \\
Ocular motility & Tumour \\
& Vascular \\
\hline
\end{tabular}

diagnosis are purposely limited to force choice. Table II shows the allowed entries for anatomical localisation and pathological diagnosis. The list of clinical diagnoses may be easily extended at the time of data entry if the list contains no appropriate diagnosis.

Patients are classified according to a combination of broad categories (anatomical localisation and pathological diagnoses) and specific categories (clinical diagnoses). Combining selection criteria for these categories allows the formation of many more categories. For instance, selection criteria in anatomy and pathology could be combined to pick out all patients with ocular motility problems due to demyelination (all cases of multiple sclerosis with ocular motility defects). Another example would be to combine selection criteria in anatomical localisation and clinical diagnosis to pick out patients with sarcoid who have an optic nerve lesion. Combining selection criteria in all three classification groups could be used to pick out patients with ocular inflammation due to sarcoid.

Judicious use of combinations of selection criteria can be used to find patients who may have been misclassified or who may be hidden away in a very specific diagnosis which does not come readily to mind. For instance, data on all patients with heredofamilial optic atrophy, whatever their precise clinical diagnosis, could be retrieved by selecting optic nerve for anatomical localisation and genetic for pathological diagnosis.

\section{Method}

A user friendly program was written with the dbase III plus package ${ }^{56}$ for a conventional IBM compatible computer with a 20-megabyte hard disc. The system created is entirely menu driven, having instructions, on screen, on how to proceed at every stage. There is no need for the user to resort to either the dbase III assist menu or have any knowledge of the dbase III language.

Consistency of data input is vital for correct data retrieval, and the program incorporates extensive validation of data input. The picture function $^{6}$ and picture template option ${ }^{6}$ are used to restrict entries to upper case letters and to provide punctuation for initials. For entries other than names and hospital numbers the computer accepts only entries that match with stored acceptable lists. If the computer rejects an entry, the user is presented with a list of acceptable entries. Extension of these lists of acceptable entries can be done at the time of patient data entry for the clinical diagnosis. Extension of the lists of anatomical localisation and pathological diagnoses is made more difficult in order to keep these two lists small, forcing choice and preventing duplication.

An opening menu provides the user with the options which include adding new patient records, editing previous patient records, searching the patient database file, and copying the database files on to a floppy disc. A customised and more friendly screen has been produced for data entry using the modify screen ${ }^{6}$ command. The program uses 11 public variables $^{6}$ corresponding to each field in the patient's record database file. Data are entered into these public variables, and validation is performed, before they are entered into the patient's record database. This system means that the delete ${ }^{6}$ and pack $^{6}$ commands are not used in the program and removes the hazard of an inexperienced user aborting the program in the middle of a pack command, when all data may be lost. ${ }^{7}$ The comparison operator ${ }^{6}$ is used to validate the entries, for sex and district, for which only a few entries are allowed. For entries for consultants, anatomical site of lesion, pathological process, and clinical diagnosis four database files ${ }^{6}$ are used to store lists of acceptable entries. Validation of data entry is then performed by matching with values in the appropriate database files.

The database files for allowed values of anatomy, pathology, and diagnosis are indexed ${ }^{6}$ to allow faster searching of them with the find ${ }^{6}$ command rather than the slower locate ${ }^{6}$ command. The user can search the patient record database for a given name or hospital number. He can also select outpatient records by entering selection criteria for lower and upper age limits, limits for date of admission, sex, district, consultant, anatomy, pathology, and diagnosis. Values entered into this combination of selection criteria then provide a filter for selecting outpatient records. If a selection criterion is left blank, then no selection is made on that field.

The selection criteria are joined by the 'and' relation. ${ }^{6}$ To prevent the user escaping to the dot prompt, on escape ${ }^{6}$ and on error ${ }^{6}$ are used to place the user with the opening menu. This is done in preference to using set escape off, ${ }^{6}$ as there may be occasions when the user wants to escape from a particular part of the program.

In theory an error will never be invoked by the program, as the program should be able to deal with all possible errors by the user. The user is encouraged to copy the database files on to a floppy disc in case the data are lost from the hard disc. Each patient record requires 306 bytes of memory.

\section{Discussion}

The program that has been developed is now in operation with the Medical Eye Unit at St Thomas's Hospital. It produces a very full description of every case, and it has proved to be user friendly. Data entry is simple and data validation has been effective. Searching of the 
data base has been rapid, specific, and complete.

We should welcome the development of similar data bases in other centres, so that exchange of records between centres could be easily achieved, which would allow access to many more patient records. A compatible national data base would allow interested clinicians to compile large lists of patients and aggregate rare cases for future research.

To this end we should be happy to supply the program written to other users. The user would require an IBM compatible machine and for copyright reasons must be in possession of the dbase III program. The user must register under the Data Protection Act, which is simply done with the appropriate hospital administrators.

1 Iwach AG, Eliason JA. Personal computers and relational databases in an eye clinic and pathology laboratory. Ophthalmology 1988; 95 (suppl): 1-5.

2 World Health Organisation. International classification of diseases. 9th revision. Geneva: World Health Organisation,

3 Taylor HR, Piro PA, Guyton DL. A classification and coding system for information retrieval on diagnosis and therapy. Ophthalmology 1983; 90: 1254-7.

4 Miller KM, Wisnicki J, Buchman JP, et al. The Wilmer information system. Ophthalmology 1988; 95: 403-9.

Ingalsbe L. Business application software for the IBM PC. Columbus: Merrill, 1987: D-2 to D-112.

6 Simpson A. Dbase III plus, programmer's reference guide. San

Francisco: Sybex, 1987 .
7 Jenkins D. Dbase, tips and tricks. New Jersey: Hayden, 1986: 24. 